\title{
ESTADO NUTRICIONAL E CARACTERÍSTICAS DE CRESCIMENTO DO ABACAXIZEIRO 'JUPI' CULTIVADO EM LATOSSOLO AMARELO DISTRÓFICO EM FUNÇÃO DA ADUBAÇÃO COM NPK
}

\author{
Nutritional status and growth caracteristics of pineapple in dystrophic yellow latosol \\ cultivated in fucntion of npk fertilization
}

\author{
Ruimário Inácio Coelho ${ }^{1}$, José Carlos Lopes ${ }^{1}$, Almy Júnior Cordeiro de Carvalho², \\ José Augusto Teixeira do Amaral ${ }^{1}$, Frederico de Pina Matta ${ }^{1}$
}

\begin{abstract}
RESUMO
Objetivou-se, neste trabalho, avaliar o efeito da aplicação da fórmula 20:05:20 de NPK no crescimento das folhas e frutos e no estado nutricional do abacaxizeiro. Foram utilizadas mudas do tipo filhote do abacaxizeiro 'Jupi', com peso médio de $250 \mathrm{~g}$, plantadas em vasos contendo $13 \mathrm{dm}^{3}$ de Latossolo Amarelo Distrófico. Os tratamentos consistiram de 00; 31; 62; $93 \mathrm{e} 124 \mathrm{~g}$ de NPK, na proporção de 20:05:20, aplicados em três parcelamentos de 20\%, 40\% e 40\% da dose aos 45, 120 e 240 dias, após o plantio. O delineamento experimental utilizado foi o de blocos casualizados, com quatro repetições. O peso médio do fruto foi influenciado pela dose de adubo, com valor significativamente maior para peso do fruto sem coroa, na dosagem de $93 \mathrm{~g}$ de adubo. Com a elevação das doses de adubo os teores de brix e acidez titulável aumentaram, enquanto a relação brix/acidez titulável apresentou redução.
\end{abstract}

Termos para indexação: Ananas comosus, abacaxi, qualidade do fruto, folha ' $\mathrm{D}$ ', nutrição.

\section{ABSTRACT}

The aim of this work was to evaluate the effects of NPK on the growth of leaves and fruits and nutritional status of pineapple. Slips of pineapple 'Jupi', with weight of $250 \mathrm{~g}$ were planted in vessel with $13 \mathrm{dm}^{3}$ of dystrophic Yellow Latosol. The treatment consisted of 00;31; 62; 93 e $124 \mathrm{~g}$ of NPK (20:05:20) divided in three applications (20\%, 40\% and 40\%) of the dose after 45, 120 and 240 days. The experiment was carried out in a randomized block outline, with four replications. The mean weight of the fruit was influenced by NPK doses. The highest fruit weight was obtained in the fruit without crown and fertilization of NPK with $93 \mathrm{~g}$. With the increase of doses of NPK, the brix tenors and total titled acidity increased while the relation brix/titled acidity decreased.

Index terms: Ananas comosus, pineapple, fruit quality, 'D' leaf, nutrition.

(Recebido em 6 de junho de 2006 e aprovado em 3 de abril de 2007)

\section{INTRODUÇÃO}

No ano de 2004, o Brasil ocupou posição de destaque entre os países produtores de abacaxi, com uma produção de 1.477.299 mil frutos em 59.163 hectares. Nesse ano, as variedades mais cultivadas foram Pérola e Smooth Cayenne (IBGE, 2006).

O abacaxizeiro é uma das principais espécies frutíferas exploradas no Espírito Santo, tradicional produtor da cultivar Pérola. Em 1996, a área colhida foi de 5.181 ha, com uma produção de 89.017 mil frutos. Porém, nos últimos anos, esse Estado vem apresentando redução na área colhida, tendo atingido, no ano de 2004, apenas 1.530 hectares com uma produção de 31.464 mil frutos (IBGE, 2006).
A produtividade e a qualidade do fruto são questões bastante complexas, pois envolvem múltiplos fatores, entre os quais a cultivar e as condições ambientais. Esses dois fatores assumem importância fundamental e torna-se necessário que a eles se associem práticas culturais adequadas, assegurando assim, o possível sucesso da cultura.

Giacomelli (1982) afirma que o tamanho dos frutos é uma das principais preocupações de quem inicia uma plantação de abacaxizeiro. Essa característica influencia diretamente no preço do fruto, pois quando o peso do fruto está dentro dos padrões, pode proporcionar maior retorno ao produtor.

\footnotetext{
'Engenheiros Agrônomos, Doutores, Professores - Departamento de Produção Vegetal/DPV - Centro de Ciências Agrárias/CCA - Universidade Federal do Espírito Santo/UFES - Alto Universitário, s/n - Cx. P. 16 - 29500-000 - Alegre, ES - ruimario@cca.ufes.br; jclopes@cca.ufes.br; jata@cca.ufes.br; fpmatta@cca.ufes.br

2Engenheiro Agrônomo, Doutor, Professor - Laboratório de Fitotecnia/LFIT - Universidade Estadual Norte Fluminense/UENF - Aveniuda Alberto Lamego, 2000, Horto - Parque Califórnia - 28013-602 - Campos dos Goitacazes, RJ - almy@uenf.br
} 
A adubação é um dos fatores responsáveis pela qualidade dos frutos, principalmente em relação ao peso alcançado. Malavolta (1982) afirma que as exigências do abacaxizeiro em nutrientes obedecem à seguinte ordem decrescente de macronutrientes: K, N, Ca, Mg, S e P, e de micronutrientes: $\mathrm{Cl}, \mathrm{Fe}, \mathrm{Mn}, \mathrm{Zn}, \mathrm{Cu}$ e B.

Tay (1975), adubando o abacaxizeiro com NPK, observou que o nitrogênio aumentou o peso e o tamanho dos frutos; porém reduziu a acidez e o teor de sólidos solúveis da polpa. O potássio aumentou o peso e tamanho do fruto, contudo a maior contribuição desse nutriente foi na elevação nos teores de acidez e dos sólidos solúveis. Resultados semelhantes foram observados por Choairy \& Fernandes (1981), Paula et al. (1991) e Teixeira et al. (2002). O emprego, em adubação, da fórmula comercial 20-05-20 (NPK), aplicada nas lavouras de abacaxizeiro nos municípios de Itapemirim e Marataízes, e a inexistência de pesquisas referentes ao assunto, motivou a execução deste trabalho, que se propôs avaliar o estado nutricional e qualidade do fruto do abacaxizeiro 'Jupi', em resposta à adubação com diferentes doses dessa fórmula NPK.

\section{MATERIAL E MÉTODOS}

O experimento foi realizado na Área Experimental do Centro de Ciências Agrárias da Universidade Federal do Espírito Santo, localizada no município de Alegre, situado a 250 metros de altitude, $20^{\circ} 45^{\prime} 48^{\prime \prime}$ de latitude Sul e longitude $41^{0} 31^{\prime} 57^{\prime \prime}$ WG. O clima da região é classificado como AW - clima tropical chuvoso com estação seca no inverno pelo sistema de Köppen, em que no local, a temperatura no mês mais quente é superior a $25^{\circ} \mathrm{C}$ e a do mês mais frio é inferior a $20^{\circ} \mathrm{C}$. A temperatura média anual é de $23,1^{\circ} \mathrm{C}$, com precipitação média anual de $1166 \mathrm{~mm}$.

As mudas tipo filhote da cultivar Jupi, com peso médio de $250 \mathrm{~g}$, foram plantadas em vasos contendo 13 $\mathrm{dm}^{3}$ de Latossolo Amarelo Distrófico coeso típico (EMBRAPA, 1999), retirado na camada de 0 a $20 \mathrm{~cm}$ de profundidade, em uma área cultivada com abacaxizeiro, localizada no município de Itapemirim. Na época, o solo apresentava as seguintes características químicas: $\mathrm{pH}=$ 5,$0 ; \mathrm{P}=1,0 \mathrm{mg} \mathrm{dm}^{-3} ; \mathrm{K}=0,03 \mathrm{cmol}_{\mathrm{c}} \mathrm{dm}^{-3} ; \mathrm{Ca}^{2+}=1,15 \mathrm{cmol}$

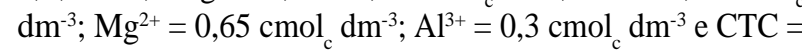
$4,7 \mathrm{cmol} \mathrm{dm}^{-3}$.

$\mathrm{O}^{\mathrm{c}}$ delineamento experimental utilizado foi o de blocos casualizados, com cinco tratamentos e quatro repetições. Cada parcela foi constituída de cinco plantas. Os tratamentos consistiram de 00; 31; 62; 93 e 124 g planta $^{-1}$ da fórmula 20:05:20 (NPK), quantidades fracionadas em três parcelas de $20 \%, 40 \%$ e $40 \%$ da dose aos 45,120 e 240 dias após o plantio.

Durante o período de realização do experimento, o substrato foi mantido com umidade de aproximadamente $50 \%$ da capacidade máxima de embebição, por meio de pesagens periódicas dos vasos e adição de água por meio de rega.

Para a avaliação do desenvolvimento vegetativo das plantas foi empregado o método da Massa Foliar Teórica (MFT), segundo a equação MFT $=($ D4 x N2) + (D6 x N4) + (D8 x N6) + (D10 x N8), em que: D4, D6, D8 e D10 correspondem aos pesos da folha ' $D$ ' obtidos, respectivamente, aos $4,6,8$ e 10 meses após o plantio, enquanto que N2, N4, N6 e N8 correspondem aos números de folhas emitidas nos dois primeiros meses, entre o quarto e o sexto meses e entre o sexto e o oitavo meses após o plantio, respectivamente (GIACOMELLI, 1982). Para as análises da composição mineral das plantas, utilizou-se a folha ' $D$ ' colhida aos dez meses após o plantio. No laboratório, as folhas foram limpas com algodão umedecido em água desionizada. A seguir, as folhas foram secas em estufa de circulação forçada de ar, a $70^{\circ} \mathrm{C}$, por 72 horas. Depois de secas, as amostras de cada tratamento foram moídas em moinho tipo Wiley, com peneira de 20 mesh e armazenadas em frascos hermeticamente fechados.

Para a determinação dos nutrientes no material moído, foram pesadas duas amostras de cada tratamento, para se proceder, respectivamente, às digestões sulfúricas $\left(\mathrm{H}_{2} \mathrm{SO}_{4}\right.$ e $\left.\mathrm{H}_{2} \mathrm{O}\right)$ e a nitro-perclórica $\left(\mathrm{HNO}_{3}\right.$ e $\left.\mathrm{HClO}_{4}\right)$. As amostras provenientes da digestão sulfúrica foram utilizadas nas análises dos teores de nitrogênio, e as de digestão nitro-perclórica nas análises dos teores de $\mathrm{P}, \mathrm{K}$, $\mathrm{Ca}, \mathrm{Mg}$. O K foi dosado por espectrofotometria de emissão atômica; o $\mathrm{P}$ foi determinado, colorimétricamente, pelo método do molibdato; o $\mathrm{Ca}, \mathrm{Mg}, \mathrm{Mn}, \mathrm{Fe}, \mathrm{Cu}$ e $\mathrm{Zn}$, por espectrofotometria de absorção atômica. $\mathrm{O} N$ foi dosado pelo método de Nessler (JACKSON, 1965). Os resultados foram apresentados em $\mathrm{g} \mathrm{kg}^{-1}$ do nutriente na massa seca da folha.

Os frutos foram colhidos em janeiro e fevereiro de 2001, sendo imediatamente pesados com e sem a coroa, obtendo-se a massa fresca dos frutos com (MFC) e sem (MFSC) a coroa. Feito isso, os frutos foram descascados manualmente e o suco foi extraído por intermédio de um espremedor manual tipo prensa. A seguir foram determinados os teores de sólidos solúveis totais (\%Brix), por refratometria, utilizando-se um refratômetro de campo, e a acidez titulável total (ATT) foi determinada por titulação, de $10 \mathrm{ml}$ do suco, com $\mathrm{NaOH}$ a $0,1 \mathrm{~N}$, usando-se uma solução alcoólica de fenolftaleína a $1 \%$ como indicador, 
sendo os resultados expressos em porcentagem de ácido cítrico (INSTITUTO ADOLFO LUTZ, 1985).

Os dados observados foram submetidos à análise de variância, em seguida aplicada análise de regressão polinomial, utilizando-se o software estatístico SAEG (Sistema para Análises Estatísticas - UFV), versão 9.0 (EUCLYDES, 2004). A escolha do modelo foi baseada na significância da regressão testada pelo teste $\mathrm{F}$ e dos coeficientes testados pelo teste t de Student, considerando um nível aceitável de até $5 \%$ de probabilidade.

\section{RESULTADOS E DISCUSSÃO}

Com doses crescentes da fórmula comercial 20-0520 (Figura 1) o crescimento do abacaxizeiro 'Jupi', expresso pela Massa Foliar Teórica (MFT), aos 10 meses após o plantio, apresentou resposta significativa de comportamento quadrático, em função da adubação. $\mathrm{O}$ maior valor para MFT $(711,775 \mathrm{~g})$ foi estimado para a dose de 74,54 g de adubo.

Em função das doses de adubo, ainda na Figura 1, observa-se o efeito significativo de resposta quadrática para massa fresca dos frutos, com e sem coroa. O maior valor para massa média de fruto sem coroa $(1199,96 \mathrm{~g})$ foi estimado pelo modelo para dose de $98,77 \mathrm{~g}_{\mathrm{g}}$ planta $^{-1}$. Paula et al. (1991) observaram efeito significativo da interação N $\mathrm{x} \mathrm{K}$ para o peso médio do fruto sem coroa, sendo o maior peso estimado (1.298 g) com $15 \mathrm{~g}$ de N planta ${ }^{-1}$ e $722 \mathrm{Kg} \mathrm{ha}^{-1}$ de $\mathrm{K}_{2} \mathrm{O}$. Resultados semelhantes também foram observados por Bezerra et al. (1981), Choairy \& Fernandes (1981) e Selamat \& Ramlah (1993).

O brix e a acidez titulável dos frutos foram influenciados pela dose de adubo e mostraram uma resposta quadrática (Figura 1). O maior valor estimado pelo modelo foi de $16,18 \%$ de Brix para dose de 65,64 g planta $^{-1}$ de adubo. Para brix e acidez do fruto, as respostas ao $\mathrm{N} \mathrm{e}$ ao K foram observadas por Paula et al. (1991), Spironello et al. (2004) e Teixeira et al. (2002), que encontraram uma correlação negativa para $\mathrm{N}$ e positiva para o K. Tais resultados mostrados diferiram parcialmente de Selamat \& Ramlah (1993). Esses últimos autores registraram decréscimos na porcentagem de sólidos solúveis totais com incremento do $\mathrm{K}$ na adubação. A relação brix/acidez, que confere sabor ao fruto, apresentou redução com a dose crescente de adubo utilizada, podendo-se atribuir o decréscimo à maior elevação da acidez em relação ao brix, uma vez que apresentaram respostas semelhantes (Figura 1).

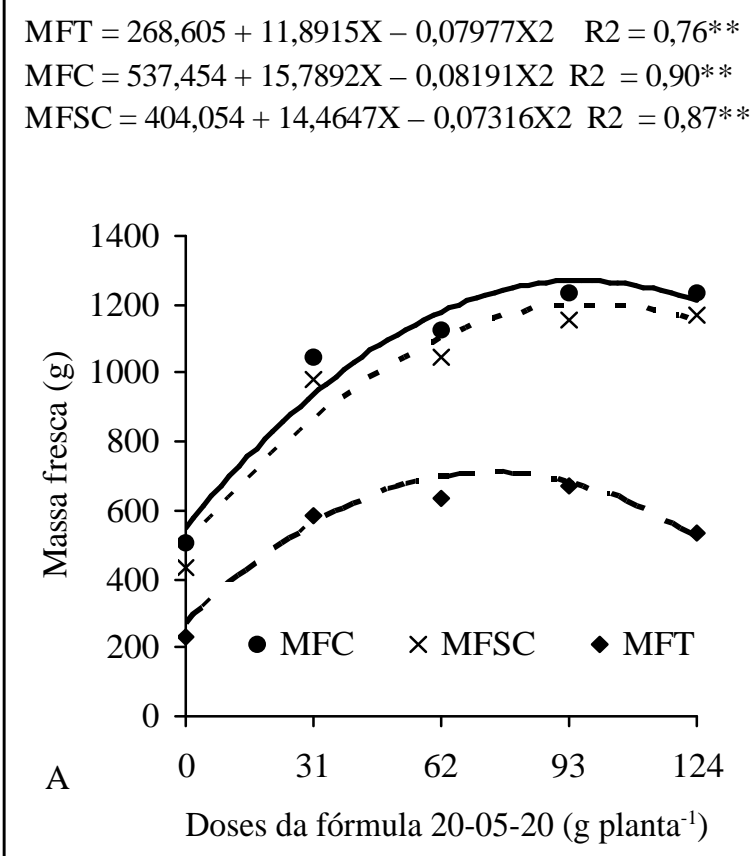

$\%$ Brix $=14,3+0,0544 X^{* *} *_{-} 0,00041 \mathrm{X} 2 * * \mathrm{R} 2=0,61 * *$
$\% \mathrm{ATT}=0,5661+0,00256 \mathrm{X}^{* *}-0,00001 \mathrm{X} 2 * * \mathrm{R} 2=0,54 * *$
Razão brix/acidez $=27,4105-0,04686 \mathrm{X}^{* *} \mathrm{R} 2=0,52 * *$

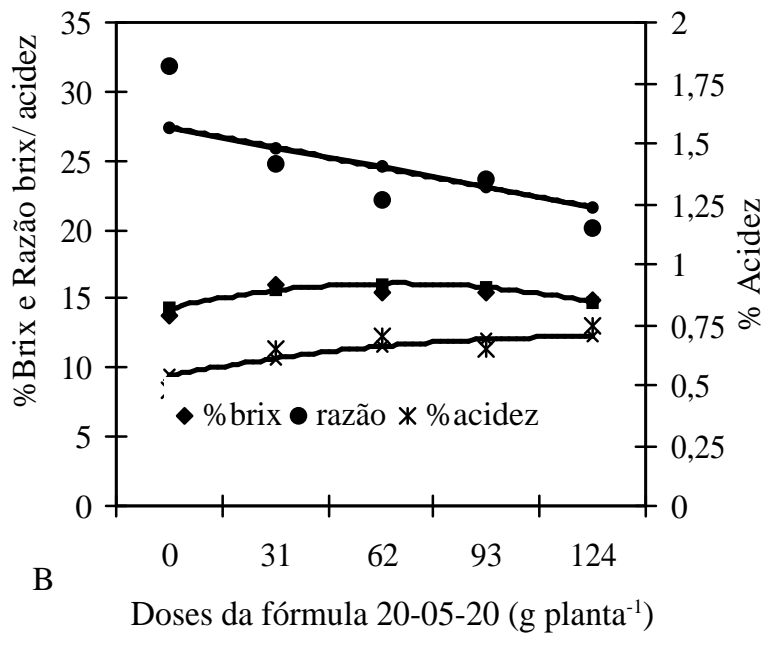

Figura 1 - Massa Foliar Teórica (MFT), Massa Fresca do Fruto com Coroa (MFC), Massa Fresca do Fruto sem Coroa (MFSC) (A) e porcentagem de Brix e de Acidez (ATT) no suco dos frutos e Razão (brix/acidez) (B), do abacaxizeiro 'Jupi', em função da dose do adubo comercial 20-05-20 (N-P-K). Alegre-ES, 2005. 
Quanto ao estado nutricional das plantas, as análises de tecidos foliares demonstraram efeito da adubação com doses crescentes da fórmula 20-05-20, nos teores dos macronutrientes N, P, K, Ca e Mg (Figura 2).

Em função da dose, na Figura 2, observou-se o aumento linear da concentração de Nitrogênio nos tecidos foliares do abacaxizeiro 'Jupi'. Nessa pesquisa, a faixa de teores foliares de 15 a $17 \mathrm{~g} \mathrm{~kg}^{-1}$, considerada adequada para a cultura (QUAGGIO et al., 1996), foi alcançada com doses de adubo superiores a $89 \mathrm{~g} \mathrm{planta}^{-1}$ (Figura 2). Porém, essa dose ficou abaixo daquelas citadas como adequadas por Haag et al. (1963) e Malavolta et al. (1997). Em função da dose de adubo, Veloso et al. (2001) observaram que o fornecimento de $12 \mathrm{~g} \mathrm{planta}^{-1}$ de $\mathrm{N}$ proporcionou teor foliar de $12,9 \mathrm{~g} \mathrm{~kg}^{-1}$, à semelhança dos resultados alcançados nessa pesquisa.

As doses crescentes de adubo proporcionaram aumento nos teores foliares de potássio, com resposta quadrática (Figura 2). O maior teor de $\mathrm{K}$ nos tecidos foliares $\left(32,77 \mathrm{~g} \mathrm{~kg}^{-1}\right)$, foi estimado pelo modelo, com a dose de $105,7 \mathrm{~g} \mathrm{planta}^{-1}$. Porém, em todas as doses de adubo estudadas, os teores foliares de $\mathrm{K}$ estiveram sempre dentro ou acima da faixa (20 a $22 \mathrm{~g} \mathrm{~kg}^{-1}$ ), faixa essa considerada adequada para a cultura, como foi citado por Boaretto et al. (1999) e Quaggio et al. (1996).

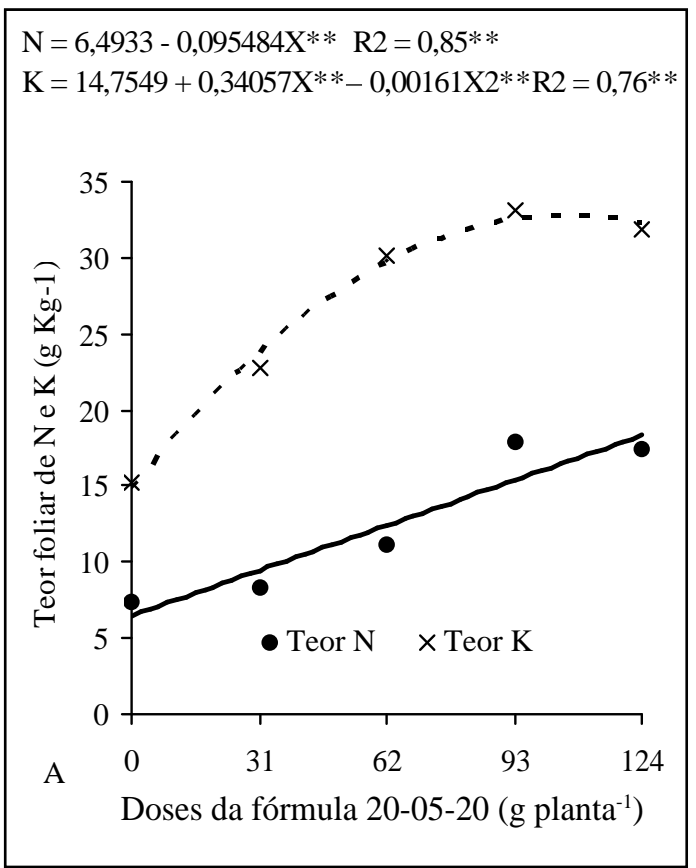

Na Figura 2, em função das doses de adubo utilizadas, observou-se aumento linear nos teores foliares de P. Em todos os tratamentos em que foi utilizado adubo, os teores foliares de $\mathrm{P}$ estiveram entre 0,9 e $1,32 \mathrm{~g} \mathrm{~kg}^{-1}$, faixa considerada adequada por Quaggio et al. (1996). Em pesquisa com parcelamento da adubação com NPK e utilizando a cultivar Smooth Cayenne, resultados semelhantes foram observados por Teixeira et al. (2002).

Com o aumento da dose de adubo, a avaliação do estado nutricional das plantas, por meio da análise de tecidos foliares, mostrou uma redução nos teores de $\mathrm{Ca}$ e $\mathrm{Mg}$, num modelo linear de primeiro e segundo graus, respectivamente (Figura 2). Resultados que, provavelmente, estão associados ao efeito de diluição dos elementos minerais nas folhas ocasionado pelo incremento no crescimento das plantas (Figura 1) e/ou à presença do $\mathrm{K}$ na adubação, ocasionando possíveis competições entre o $\mathrm{K}$ e o $\mathrm{Ca}$ e o $\mathrm{Mg}$ no processo de absorção pelas raízes. Paula et al. (1991) e Veloso et al. (2001) observaram que a aplicação de altas doses de $\mathrm{K}$ reduz o conteúdo de Ca e $\mathrm{Mg}$, nas folhas do abacaxizeiro.

De acordo com Quaggio et al. (1996), para o Ca e $\mathrm{Mg}$, os teores foliares registrados nas análises em todos os tratamentos ficaram abaixo das faixas adequadas de nutrição para o abacaxizeiro.

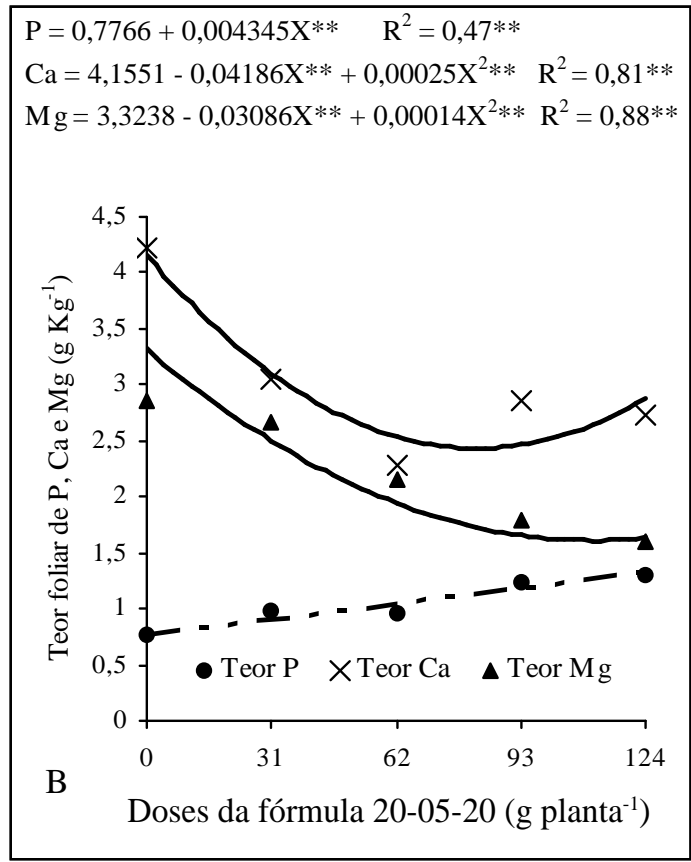

Figura 2 - Teores de N, K (A) e de P, Ca e $\mathrm{Mg}(\mathbf{B})$ na matéria seca da folha 'D' do abacaxizeiro 'Jupi', em função das doses do adubo comercial 20-05-20 (N-P-K). Alegre-ES, 2005. 
Os teores de $\mathrm{Cu}, \mathrm{Zn}$, Fe e Mn no tecido foliar, em função das doses da fórmula 20-05-20 encontram-se na Tabela 1. Não houve efeito dos tratamentos nos teores foliares desses nutrientes, os quais se encontram dentro ou acima das faixas citadas por Malavolta et al. (1997), como adequadas para o abacaxizeiro. Souza et al. (2002) não observaram efeito significativo da adubação potássica sobre os teores foliares de $\mathrm{Mn}, \mathrm{Cu}, \mathrm{Fe}$ e $\mathrm{Zn}$.

Tabela 1 - Influência das doses da fórmula comercial 2005-20 (N-P - K ) em g planta ${ }^{-1}$ sobre os teores foliares de $\mathrm{Cu}, \mathrm{Zn}, \mathrm{Fe}$ e Mn, em abacaxizeiro 'Jupi'. Alegre-ES, 2005.

\begin{tabular}{|c|c|c|c|c|}
\hline Doses & $\mathrm{Cu}$ & $\begin{array}{l}\mathrm{Zn} \\
\mathrm{m}\end{array}$ & $\begin{array}{r}\mathrm{Fe} \\
\mathrm{kg}^{-1} \\
\end{array}$ & Mn \\
\hline $\begin{array}{c}0 \mathrm{~g} \mathrm{planta}^{-1} \mathrm{da} \\
\text { fórmula } \\
20-05-20\end{array}$ & 11,11 & 13,38 & 152,89 & 492,43 \\
\hline $\begin{array}{l}31 \text { g planta }^{-1} \mathrm{da} \\
\text { fórmula } \\
20-05-20\end{array}$ & 12,83 & 11,70 & 154,68 & 510,87 \\
\hline $\begin{array}{l}62 \mathrm{~g} \mathrm{planta}^{-1} \mathrm{da} \\
\text { fórmula } \\
20-05-20\end{array}$ & 13,92 & 10,99 & 146,71 & 515,78 \\
\hline $\begin{array}{c}93 \text { g planta }^{-1} \mathrm{da} \\
\text { fórmula } \\
20-05-20\end{array}$ & 12,76 & 12,43 & 185,43 & 482,89 \\
\hline $\begin{array}{c}124 \text { g planta }^{-1} \mathrm{da} \\
\text { fórmula } \\
20-05-20\end{array}$ & 12,57 & 11,70 & 152,63 & 483,58 \\
\hline Média & 12,64 & 12,04 & 158,47 & 497,11 \\
\hline CV (\%) & 23,60 & 20,64 & 20,63 & 21,60 \\
\hline
\end{tabular}

${ }^{{ }^{n}}$ não significativo ao nível de $5 \%$ de probabilidade pelo Teste de F.

\section{CONCLUSÕES}

Nas condições deste experimento, pode-se afirmar que:

- o crescimento das plantas e a massa fresca dos frutos são influenciados pelas doses da formula 20-05-20;

- o aumento da dose da fórmula aumentou os teores de brix e a acidez dos frutos, porém, reduziu a relação brix/ acidez;

- doses da fórmula 20-05-20 acima de $80 \mathrm{~g}_{\text {planta }}{ }^{-1}$ proporcionaram uma nutrição adequada das plantas do abacaxizeiro para N, P e K;
- os teores foliares de $\mathrm{Ca}$ e $\mathrm{Mg}$ foram reduzidos com doses crescentes da fórmula 20-05-20.

\section{REFERÊNCIAS BIBLIOGRÁFICAS}

BEZERRA, J. E. F.; MAAZE, U. C.; SANTOS, V. F. dos; LIDERMAN, I. E. Efeito da adubação nitrogenada, fosfatada e potássica na produção e qualidade do abacaxi cv. Smooth Cayenne. Revista Brasileira de Fruticultura, Cruz das Almas, v. 3, p. 1-5, 1981.

BOARETTO, A. E.; CHITOLINA, J. C.; RAIJ, B. V.; SILVA, F. C. da; TEDESCO, M. J.; CARMO, C. A. F. de S. do. Amostragem, acondicionamento e preparação das amostras de plantas para análise química. In: SILVA, F. C. da (Org.). Manual de análises químicas de solos, plantas e fertilizantes. Brasília, DF: Embrapa Comunicação para Transferência de Tecnologia, 1999. p. 49-74.

CHOAIRY, S. A.; FERNANDES, P. D. Adubação NPK do abacaxizeiro: dose e modo de aplicação. In: CONGRESSO BRASILEIRO DE FRUTICULTURA, 6., 1981, Recife.Anais... Recife: SBF, 1981. v. 1, p. 67-76.

EMPRESA BRASILEIRA DE PESQUISA AGROPECUÁRIA. Centro Nacional de Pesquisa de Solos. Sistema brasileiro de classificação de solos. Rio de Janeiro, 1999. $412 \mathrm{p}$.

EUCLYDES, R. F. Sistema para análises estatísticas. Versão 9.0. Viçosa: FUNARBE; UFV, 2004.

GIACOMELLI, E. J. Expansão da abacaxicultura no Brasil. Campinas: Fundação Cargill, 1982. 79 p.

HAAG, H. P.; ARZOLA, S.; MELLO, F. A. F.; BRASIL SOBRINHO, M. O. C.; OLIVEIRA, E. R.; MALAVOLTA, E. Estudo sobre alimentação mineral do abacaxi. Anais da Escola Superior de Agricultura "Luiz de Queiroz", Piracicaba, v. 20, p. 34-40, 1963.

INSTITUTO ADOLFO LUTZ. Normas analíticas do Instituto Adolfo Lutz. 3. ed. São Paulo, 1985. v. 1, 533 p.

INSTITUTO BRASILEIRO DE GEOGRAFIA E ESTATÍSTICA. Levantamento sistemático da produção agrícola 2006. Disponível em: <http:// www.sidra.ibge.gov.brbda/agric/default>. Acesso em: 24 mar. 2006. 
JACKSON, M. L. Soil chemical analysis. New Jersey: Prentice Hall, 1965. 498 p.

MALAVOLTA, E. Nutrição mineral e adubação do abacaxizeiro. In: SIMPÓSIO BRASILEIRO SOBRE ABACAXICULTURA, 1., 1982. Jaboticabal. Anais... Jaboticabal: Faculdade de Ciências Agrárias e Veterinárias, 1982. p. 121-153.

MALAVOLTA, E.; VITTI, G. C.; OLIVEIRA, S. A. Avaliação do estado nutricional das plantas: princípios e aplicações. 2. ed. Piracicaba: Potafos, 1997. 319 p.

PAULA, M. B. de; CARVALHO, V. D. de; NOGUEIRA, F. D.; SOUZA, L. F. da S. Efeito da calagem, potássio e nitrogênio na produção e qualidade do fruto do abacaxizeiro. Pesquisa Agropecuária Brasileira, Brasília, v. 26, n. 9 , p. $1337-1343,1991$

QUAGGIO, J. A.; RAIJ, B. V.; PIZA JUNIOR, C. T. Frutíferas. In: RAIJ, B. van et al. (Eds.). Recomendação de adubação e calagem para o Estado de São Paulo. 2. ed. Campinas: IAC, 1996. p. 121-5. (Boletim técnico, 100).

SELAMAT, M. M.; RAMLAH, M. The response of pineapple cv gandul to Nitrogen, Phosphorus, and Potassium on peat soil in Malaysia. Acta Horticulturae, Amsterdam, v. 334, p. 247-254, 1993.
SOUZA, L. F. da S.; GONÇALVES, N. B.; CALDAS, R. C.; SOARES, A. G.; MEDINA, V. M. Influência da adubação potássica nos teores foliares de nutrientes do abacaxizeiro 'Pérola'. In: CONGRESSO BRASILEIRO DE FRUTICULTURA, 17., 2002, Belém. Anais... Belém: SBF, 2002. CD-ROM.

SPIRONELLO, A.; QUAGGIO, J. A.; TEIXEIRA, L. A. J.; FURLANI, P. R.; SIGRIST, J. M. M. Pineapple yield and fruit quality affected by NPK fertilization in a tropical soil. Revista Brasileira de Fruticultura, Jaboticabal, v. 26, n. 1, p. 55-159, 2004.

TAY, T. H. Effects of nitrogen and potassium on the growth, mean fruit weight and fruit quality of pineapple. Mardi Research Bulletin, [S.1.], v. 3, p. 1-14, 1975.

TEIXEIRA, L. A. J.; SPIRONELLO, A.; FURLANI, P. R.; SIGRIST, J. M. M. Parcelamento da adubação NPK em abacaxizeiro. Revista Brasileira de Fruticultura, Jaboticabal, v. 24, n. 1, p. 219-224, 2002.

VELOSO, C. A. C.; OEIRAS, A. H. L.; CARVALHO, E. J. M.; SOUZA, F. R. S. de. Resposta do abacaxizeiro à adição de nitrogênio, potássio e calcário em Latossolo Amarelo do Nordeste Paraense. Revista Brasileira de Fruticultura, Jaboticabal, v. 23, n. 2, p. 396-402, 2001. 